\title{
Clarifying the dilemmas about inhalation techniques for dry powder inhalers: integrating science with clinical practice
}

\author{
Wahida Azouz ${ }^{\mathrm{a}}$, Henry Chrystyn ${ }^{\mathrm{a}}$ \\ a Division of Pharmacy and Pharmaceutical Sciences, School of Applied Sciences, University of Huddersfield, Huddersfield, \\ West Yorkshire, HDI 3DH, UK
}

Originally received 22nd July 2011; resubmitted 18th October 2011; revised 23rd November 2011; accepted 12th December 2011; online 15th February 2012

\begin{abstract}
This review integrates pharmaceutical science with routine clinical practice to explain why inhalation manoeuvres through a dry powder inhaler (DPI) should start with a gentle exhalation, away from the inhaler. Place the inhaler in the mouth and ensure the lips form a tight seal. This should be followed by an immediate forceful inhalation that is as fast as possible and continued for as long as the patient can comfortably achieve. Although this is universally accepted, there has been a lot of attention on inhalation flow as an indicator of adequate inspiratory effort. This has led to the wrong assumption that inhalation flows through each DPI should be the same, and that low flows through some DPIs suggest that dose delivery is impaired. Most miss the concept that inhalation flow together with the resistance of the DPI combine to create a turbulent energy which de-aggregates the formulation and provides an effective emitted dose. A low flow through a DPI with high resistance generates the same turbulent energy as fast flow with low resistance. Therefore, depending on the device, different inhalation flows are compatible with potentially effective use. Flow measurements should be a guide to train patients to inhale faster. The focus of inhaler technique training should be the use of the above generic inhalation manoeuvre.

(C) 2012 Primary Care Respiratory Society UK. All rights reserved.

W Azouz and H Chrystyn. Prim Care Respir J 2012; 21(2): 208-213

http://dx.doi.org/10.4104/pcrj.2012.00010
\end{abstract}

Keyw ords chronic obstructive pulmonary disease, dry powder inhaler, inhalation manoeuvre

\section{Introduction}

To ensure compliance with the prescribed inhaler regimen and correct inhaler administration, patients should be prescribed an inhaler that they can and will use. This is the theme of the recently published European Respiratory Society/International Society for Aesosols in Medicine (ERS/ISAM) Task Force Consensus Statement on inhaler use ${ }^{1}$, and it is consistent with the recommendations of the Aerosol Drug Management Improvement Team (ADM IT) group which were consolidated in their latest article published recently in this journal. ${ }^{2}$ These publications highlight the fact that the main problems with dry powder inhaler (DPI) use experienced by patients with asthma (children and adults) and chronic obstructive pulmonary disease (COPD) are dose preparation and exhalation before an inhalation, as well as not using a forceful inhalation. ${ }^{3.5}$ Broeders et al., ${ }^{2}$ like many others, recommend that the patient's ability to generate an appropriate inhalation flow through a DPI should be considered. However, this statement is vague and not well defined, which leads to misinterpretations about inhalation flow and DPIs.

Some COPD patients, as well as young children with asthma and (to some extent) patients with severe asthma, have a reduced inspiratory effort which leads to lower flows through the DPI, ${ }^{6}$ which is why extra attention should be directed to these patients when they use a DPI. Each type of DPI has a unique resistance to airflow, and inhalation flows through high resistance devices are naturally lower than through those with a lower resistance. ${ }^{6}$ Focusing on inhalation flow and not integrating this with the resistance of the DPI, and extending this to the resultant turbulent energy generated inside the DPI during an inhalation, has led to misunderstanding about inhalation flow.

In this review we have used a science-based approach, integrated with clinical practice, to explain why the ERS/ISAM Task Force Consensus Statement states that important points to consider with respect to the inhalation manoeuvre when using a DPI are:

\footnotetext{
* Corresponding author: Professor Henry Chrystyn, Division of Pharmacy and Pharmaceutical Sciences, School of Applied Sciences, University of Huddersfield, Huddersfield, West Yorkshire HDI 3DH, UK. Tel: +44 (0)1484 472783 Fax: +44 (0)1484 472182 E-mail: h.chrystyn@hud.ac.uk
} 
- Inhale as fast as you can

- Inhale forcefully from the start of an inhalation

- Continue the inhalation as long as possible

To assist with our explanation, we have included data on three multidose DPIs - a medium resistance DPI (Accuhaler), a medium/high resistance DPI (Turbuhaler), and a high resistance DPI (Easyhaler). Although the recommended inhalation manoeuvre ${ }^{1}$ applies to all patients who use a DPI, this review will concentrate on patients with COPD because of their reduced inspiratory effort.

We also include the scientific rationale as to why patients should exhale before an inhalation and why this exhalation should not be made through the DPI. These were also highlighted by the ERS/ISAM Task Force Consensus Statement. ${ }^{1}$

\section{Inhale as fast as you can through a DPI}

The emitted dose from an inhaler must contain drug particles $<5 \mu m$ to enable penetration into the airways.' However, it is not possible to formulate DPIs to contain particles that are so small because the formulation will have poor powder flow properties. This would cause erratic inhaler filling during manufacture and inconsistent dose metering during patient use. Poor powder flow is improved by DPI formulations that are either a mixture of drug particles and an excipient (usually lactose) or when the drug particles are formulated into spherical agglomerates. The drug particles in these formulations are too big to penetrate into the airways. After dose metering, the formulation of a DPI is broken up (de-aggregated) by a turbulent energy that is created by the interaction of the patient's inhalation flow with the resistance inside the inhaler. ${ }^{7}$ A schematic design of this process, using a cross-section of the Easyhaler, is shown in Figure 1. The deaggregation occurs in the channel that leads from the metered dose to the exit. Cross-sections of the Accuhaler and the Turbuhaler are shown in Figures $2 \mathrm{a}$ and $2 \mathrm{~b}$ and highlight where the de-aggregation occurs in these DPIs (same principle as that described in Figure 1). The turbulent energy is represented by a pressure change inside each DPI during the inhalation manoeuvre. Pressure is measured by various units (all are related) with kilopascals $(\mathrm{kPa})$ used for DPIs.

The turbulent energy inside a DPI during an inhalation is the product of the inhalation flow and the resistance of the device: ${ }^{11}$

\section{$\sqrt{ } \mathrm{P}=\mathrm{Q} \times \mathrm{R}$}

where $P$ is the turbulent energy, $Q$ is the inhalation flow and $R$ is the resistance. Hence, to achieve a set energy inside a DPI during an inhalation, if the resistance is low then the inhalation flow will be higher than that required through a DPI with a higher resistance. The resistance of reservoir multidose DPIs ranges from medium (Accuhaler) to medium/high (Turbuhaler) to high (Easyhaler).' The higher the resistance and the greater the patient's airflow obstruction, the lower will be the inhalation flow, ${ }^{6}$ but this does not necessarily translate to inadequate turbulent energy and de-aggregation during patient use when the inhalation flow is low. Furthermore, the above equation shows that the relationship between flow and the resultant

Figure 1. The de-aggregation process that occurs in a dry pow der inhaler (DPI) during an inhalation. This example shows how the interaction between the patient's inhalation and the resistance of the inhaler generates the turbulent energy to split the small drug particles from their larger carrier (in this case lactose) particles. Reproduced from Chrystyn ${ }^{8}$ with permission

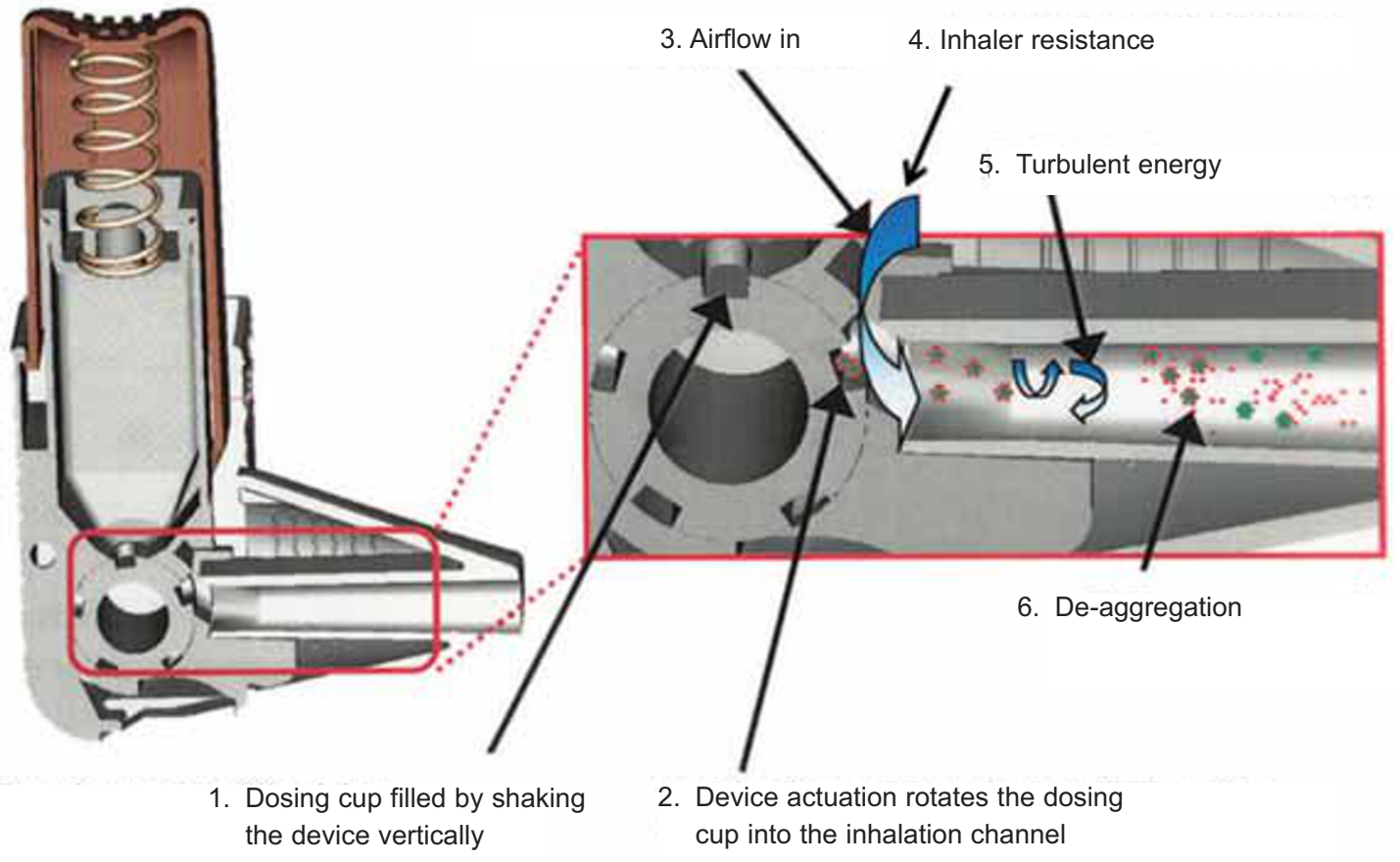


Figure 2. Cross section of (a) an Accuhaler and (b) a Turbuhaler. The area within the dashed line represents the inhalation channels where de-aggregation occurs during an inhalation. Reproduced from Prime et al. ${ }^{9}$ and Persson et al. ${ }^{10}$ with permission

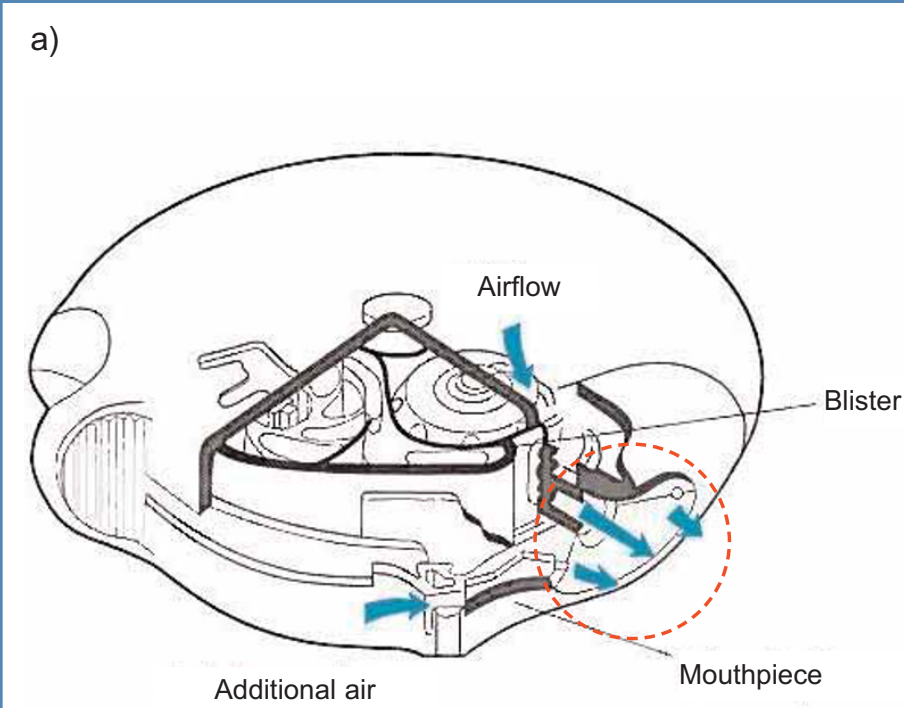

b)

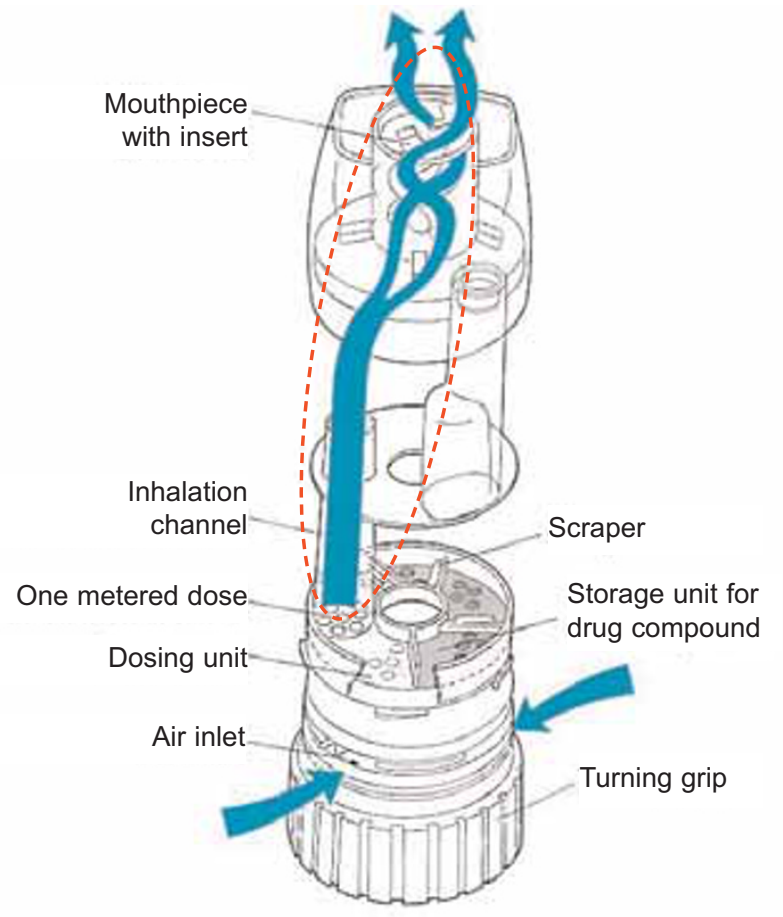

turbulent energy is not linear. The inhalation flows of patients with COPD were, as expected, reported to be faster through an Accuhaler than a Turbuhaler (111.4 vs. $76.9 \mathrm{~L} / \mathrm{min})$ but the resultant energy was the same. ${ }^{12}$ Similarly, in our COPD studies, inhalation flows through an Accuhaler, Turbuhaler and Easyhaler were in the expected order, but the maximum turbulent energy inside each device during inhalation was $3.4 \mathrm{kPa}, 2.9 \mathrm{kPa}$ and $5.8 \mathrm{kPa}$, respectively. Thus, although the flow through the Easyhaler was the lowest, the turbulent energy available to deaggregate the dose was the highest. These energies may explain the different flow-dependent dose emissions of these DPIs ${ }^{13}$ and why DPIs with a high resistance tend to provide greater lung deposition with less variability than those with a lower resistance. $^{14}$

When comparing different flows with the same DPI, the above equation explains the flow-dependent dose emission properties of DPIs. ${ }^{13}$ In the past this relationship has been misrepresented by attempting to define an optimal flow. At present there is no evidence for an optimal flow through each DPI. Instead of trying to define optimal flow, focus should be directed to the minimum flow through each DPI below which de-aggregation is significantly reduced. In vitro studies have shown that there is an inhalation flow through a DPI below which the turbulent energy is not sufficient to de-aggregate the dose efficiently. ${ }^{15}$ This study confirmed that, below $30 \mathrm{~L}$ min, the turbulent energy will not be able to de-aggregate the formulation of the Turbuhaler efficiently and there are clinical data to support this. ${ }^{16}$ It is not correct to assume that this threshold is the same for every DPI without any evidence. For DPIs with a higher resistance than the Turbuhaler, the flow corresponding to this critical threshold for turbulent energy will be slower and vice versa.

Clinical studies using the Turbuhaler ${ }^{16}$ and the Accuhaler ${ }^{17}$ have identified why the minimum effective flow through these DPIs is regarded to be $30 \mathrm{~L} / \mathrm{min}$. The Easyhaler has a higher resistance and therefore, as expected, is found to be effective ${ }^{18}$ and to provide similar lung deposition to a metered dose inhaler (MDI) attached to a spacer ${ }^{19}$ at inhalation flows $<30 \mathrm{~L} / \mathrm{min}$ (the lowest flow was $16 \mathrm{~L} / \mathrm{min}) .^{18}$ This suggests that the resultant turbulent energy generated from these low flows in this high resistance DPI was sufficient to de-aggregate the dose and highlights the fact that each type of DPI is unique. Owing to these unique properties and the different dose preparation manoeuvres, DPIs should be prescribed by brand name, not generic name. Furthermore, a generic inhaler-switching policy should not be used. It has been shown that, after switching inhalers during the prescription and dispensing process, asthma control was reduced and GP consultations increased. ${ }^{20}$ Thus, although item costs would have been reduced, the total healthcare costs would have increased.

When a forceful inhalation manoeuvre is used, most patients can achieve adequate flows through their DPI. ${ }^{14}$ However, some young children ${ }^{6,14,16,21}$ and patients with severe COPD ${ }^{6,14}$ may have problems. When checking their inhalation technique, if these patients cannot make a determined forceful inhalation through their DPI, they should be prescribed a DPI with lower resistance 
or an MDI, with or without a spacer. It has been reported that it is possible to train patients with COPD to use correct flows through a pressurised MDI rather than faster flows through a DPI. ${ }^{22}$

There is also some concern about impaired inspiratory effort during exacerbations which could translate to reduced dose emission from a DPI. This concern is usually directed to patients with COPD and young children with asthma because of their reduced inspiratory effort. In COPD this effect during acute exacerbations was not sufficient to cause concern, ${ }^{23}$ but how this reflects to real life is not known. Reduced dose delivery from all DPIs should be taken into consideration during exacerbations. Overall, the reduction in the inhalation flow achieved through a high resistance device should be less than that of a low resistance DPI. ${ }^{24}$

\section{Inhale forcefully from the start of an inhalation through a DPI}

The de-aggregation of the metered dose in a DPI occurs during the first part of the inhalation manoeuvre ${ }^{25}$ and therefore the patient should be made aware that the forceful inhalation should be from the beginning. Figure 3 describes how the peak inhalation flow (and hence the turbulent energy) can be achieved using two different inhalation manoeuvres and also shows that the de-aggregated dose leaves a multidose DPI in the first part of the inhalation, before the peak inhalation flow is achieved. ${ }^{26}$ Comparing the two inhalation profiles in Figure 3, there would be a big difference between the turbulent energy that is created

Figure 3. Inhalation flow against time proffles of two different inhalation manoeuvres through a dry powder inhaler (DPI). The two proffles have the same peak inhalation flow. The one with the steep acceleration is a forceful inhalation from the start of an inhalation (solid line) whereas the proffle with a gentler acceleration is an inhalation manoeuvre that starts slowly and gradually builds up into a flow that is as fast as possible (dashed line). Superimposed onto the proffles is the time period during which the dose is de-aggregated and emitted from the DPI. Adapted from Chrystyn \& Price ${ }^{26}$ with permission

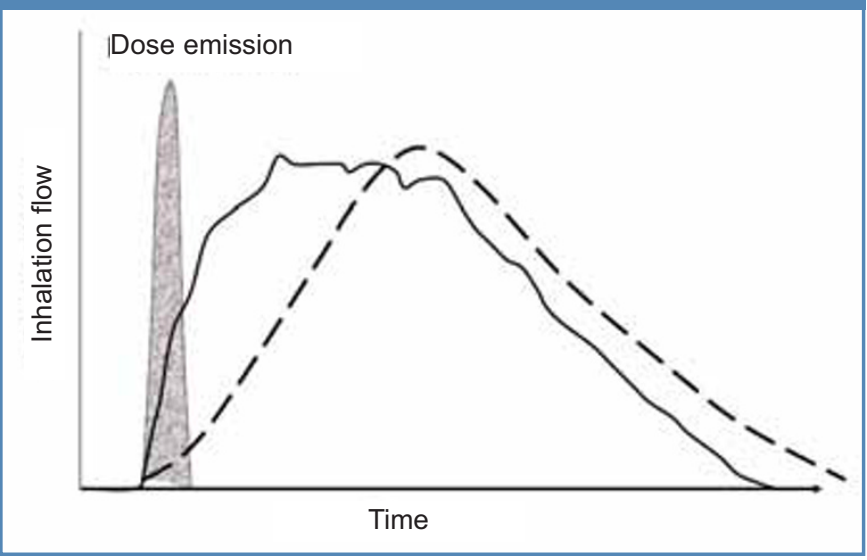

PRIMARY CARE RESPIRATORY JOURNAL www.thepcrj.org in the first part of the inhalation when de-aggregation occurs. The turbulent energy during the initial phase of an inhalation is only linked to the peak inhalation flow when the forceful inhalation starts immediately. ${ }^{27}$ The ERS/ISAM Consensus Statement therefore recommends that patients should be instructed to inhale forcefully from the beginning and should not increase the speed of their inhalation gradually. ${ }^{1}$ This again highlights the value of integrating pharmaceutical science with practice.

Not inhaling forcefully and deeply is one of the common mistakes made by patients with $\mathrm{COPD}{ }^{3-5}$ and this can be improved with training. ${ }^{9}$ It is therefore important to train patients to use a forceful inhalation from the start of their inhalation. This could be done by observation or with the help of the IN-Check Dial (Clement Clarke International, UK). The IN-Check Dial is a useful training aid ${ }^{28}$ that can be used with inhalation technique training to help patients achieve a faster inhalation flow. It should be used with a visual check that the patient is inhaling forcefully from the beginning. There is no clear clinical evidence of the optimal flow required for a DPI, so care should be used if the In-Check Dial is used as a device selection tool.

\section{Inhale for as long as you can}

Another inhalation parameter which has been mostly ignored to date is the inhalation volume. In vitro studies have shown the importance of the inhalation volume for emptying the dose from a capsule DPI. ${ }^{29}$ For this reason, the ERS/ISAM Task Force has recommended that each patient should use two separate inhalations when using a capsule DPI and that, for each DPI, the inhalation should continue for as long as possible." Deaggregation of the dose emitted from a capsule DPI also requires the recommended immediate forceful inhalation. ${ }^{1}$ Other in vitro studies have shown the significance of dose emission and inhalation volume from multidose DPIs, which is more pronounced for some ${ }^{30}$ but not sufficient to warrant two separate inhalations per dose.

Patients with COPD have low inhalation volumes when they inhale through DPIs. ${ }^{31}$ Since failing to exhale before an inhalation through a DPI is a common mistake ${ }^{3-5}$ and lung deposition is related to the extent of the exhalation, ${ }^{32}$ patients should be trained to exhale gently before they start to inhale. The increased volume will ensure better drug penetration into their airways and deposition onto the therapeutic targets in the lungs.

\section{Exhale aw ay from the DPI before an inhalation manoeuvre}

Many patients exhale through their DPIs, ${ }^{3-5}$ and this will introduce moisture into the formulation. This affects powder flow which will cause problems with dose metering and de-aggregation. ${ }^{33}$ Depending on the internal design of the DPI, some will be affected more than others. Also, exhalation after the dose has been prepared for inhalation will blow the dose out through the inhalation channels of the DPI. The patient will therefore receive 
no dose during inhalation, so patients should be trained to exhale away from their inhaler. ${ }^{1}$

\section{Dry pow der inhaler choice}

The principles and implications described above also apply to patients with asthma (children and adults) when they use a DPI. The current trend for COPD is to use inhaled long-acting bronchodilators (long-acting $\beta_{2}$-agonists and long-acting muscarinic antagonists) formulated in different DPIs. These DPIs will have different resistances, so we should not compare the different flows achieved nor focus on optimal flows. The inhaler prescribed should be one that the patient can and will use, so patient choice is more important than prescriber preference. Thus, prescribers should be aware of the different instructions about dose preparation, but the inhalation manoeuvre is generic. When using a DPI, patients should be aware of the recommended dose preparation procedures and the need to exhale away from the inhaler immediately before inhalation. Dose preparation errors are common ${ }^{3.5}$ and are likely to lead to no dose being inhaled. Those DPIs with a few simple dose preparation steps should be easy to use. Patients should also be aware of their lack of compliance because this is the most important critical error.

Guides based on inhalation flow ${ }^{1,34}$ are available for choosing inhalers, but these assume that the resistance of all DPIs is the same. These guides are useful, especially for young children with asthma and patients with severe COPD, but inhalation flow should not be used as the criterion to select the inhaler.

It is important that there is a range of therapies in the same device but, for patients with COPD, this is not possible at present. Although using more than one type of inhaler does cause problems, ${ }^{35}$ one solution would be to use DPls that have a similar resistance. Training aids are useful, ${ }^{28}$ but at present they are not sufficiently sophisticated to ensure that the inhalation manoeuvre recommended for a DPI is achieved. Such a training aid should monitor inhalation flow with respect to time and translate this into turbulent energy, acceleration rates and inhalation volumes.

\section{Conclusions}

This review has integrated pharmaceutical science with the routine use of DPIs to explain that inhalation flow through a DPI should not be considered in isolation. The review explains that the turbulent energy generated inside an inhaler is more important than inhalation flow, and it describes how this is generated during an inhalation manoeuvre. Training in inhalation technique with a focus on using a gentle exhalation followed by a forceful inhalation from the start (i.e, as fast as the patient can achieve) and continued for as long as possible should ensure that most patients can use a DPI irrespective of the resistance of the device or the severity of their chest disease. Low inhalation flows through a DPI with high resistance do not mean that patients should not be prescribed that inhaler compared with a higher flow through a DPI with less resistance. Some patients with COPD, especially those with severe disease, as well as some young children with asthma may have problems achieving the critical threshold for de-aggregation of the formulation from the inhalation flows they achieve. If these patients cannot use a forceful inhalation that begins immediately, an alternative inhaler should be prescribed (MDI with or without a spacer, Respimat for (OPD).

\section{Handling editor Sundeep Salvi}

Conflicts of interest WA has no conflicts of interest. HC has no shares in any pharmaceutical companies. He has received sponsorship to carry out studies, together with some consultant agreements and honoraria for presentation, from several pharmaceutical companies which market inhaled products including Almirall, AstraZeneca, Boehringer Ingelheim, Chiesi, GlaxoSmithKline, Innovata Biomed, Meda, Mundipharma, Orion, Teva, Truddell and UCB. Research sponsorship has also been received from grant awarding bodies (EPSRC and MRC).

Contributorship All authors contributed to the review of the literature, formulation of content and drafting the manuscript.

Funding None.

\section{References}

1. Laube BL, Janssens HM, de Jongh FHC, et al. ERS/ISAM Task Force Consensus Statement: Recommendations on what the pulmonary specialist should know about the new inhalation therapies. Eur Respir J 2011;37:1308-31. http://dx.doi.org/10.1183/09031936.00166410

2. Broeders MEAC, Vincken W, Corbetta L. Ways to improve pharmacological management of COPD: the importance of inhaler choice and inhalation technique. Prim Care Respir J 2011;20:338-43. http://dx.doi.org/10.4104/pcri.2011.00062

3. Molimard M, Raherison C, Lignot S, Depont F, Abouelfath A, Moore N. Assessment of handling on inhalers in real life: an observational study in 3811 patients in primary care. J Aerosol Med 2003;16:249-54 http://dx.doi.org/10.1089/ 089426803769017613

4. Lavorini F, Magnan A, Dubus JC, et al. Effect of incorrect use of dry powder inhalers on management of patients with asthma and COPD. Respir Med 2008;102:593-604. http://dx.doi.org/10.1016/j.rmed.2007.11.003

5. Melani AS, Bonavia M, Cilenti $\mathrm{V}$, et al., on behalf of the Gruppo Educazionale Associazione Italiana Pneumologi Ospedalieri (AIPO). Inhaler mishandling remains common in real life and is associated with reduced disease control. Respir Med 2011;105:930-8. http://dx.doi.org/10.1016/j.rmed.2011.01.005

6. Chrystyn $\mathrm{H}$. Effects of device design on patient compliance: comparing the same drug in different devices. In: Dalby RN, Byron PR, Peart J, Suman JD, Young PM, eds. Respiratory Drug Delivery Europe 2009. Volume 1. Richmond, VA, USA: Davis Healthcare International Publishing, Virginia Commonwealth University, 2009, p.10516.

7. Chrystyn $\mathrm{H}$. Is inhalation rate important for dry powder inhalers? Using the In-Check Dial to identify these rates. Respir Med 2003;97:181-18. http://dx.doi.org/ 10.1053/rmed.2003.1351

8. Chrystyn H. Closer to an 'Ideal Inhaler' with the Easyhaler: An Innovative Dry Powder Inhaler. Clin Drug Invest 2006;26(4):175-83. http://dx.doi.org/10.2165/00044011-200626040-00001

9. Prime D, Slater AL, Haywood PA Smith IJ. Assessing dose delivery from the Flixotide Diskus Inhaler - a multidose powder inhaler. Pharmaceutical Technology Europe 1996;8(3):23-34.

10. G Persson, E Gruvstad, E Stahl. A new multiple dose powder inhaler, (Turbuhaler), compared with a pressurized inhaler in a study of terbutaline in asthmatics. Eur Respir J 1988;1:681-4

11. Clark AR, Hollingworth AM. The relationship between powder inhaler resistance and peak inspiratory conditions in healthy volunteers: implications for in vitro testing. J Aerosol Med 1993;6:99-110. http://dx.doi.org/10.1089/jam.1993.6.99

12. Broeders MEAC, Molama J, Hop WCJ, Folgering HTM. Inhalation profiles in asthma and COPD patients: reproducibility and effect of instruction. J Aerosol Med 2003;16:131-41. http://dx.doi.org/10.1089/089426803321919898 
13. Palander A, Mattila T, Karka M, M uttonen E. In-vitro comparison of three salbutamolcontaining multidose dry powder inhalers. Clin Drug Invest 2000;20:25-33. http://dx.doi.org/10.2165/00044011-200020010-00004

14. Borgstrom $L$. On the use of dry powder inhalers in situations perceived as constrained. J Aerosol Med 2001;14:281-7. http://dx.doi.org/10.1089/089426801316970231

15. Nadarassan DK, Assi KH, Chrystyn H. Aerodynamic characteristics of a dry powder inhaler at low inhalation flows using a mixing inlet with an Andersen Cascade Impactor. Eur J Pharm Sci 2010;39:348-54. http://dx.doi.org/10.1016/ j.ejps.2010.01.002

16. Pedersen $S$, Hansen R, Fuglsang $G$. Influence of inspiratory flow rate upon the effect of a Turbuhaler. Arch Dis Child 1990;65:308-19. http://dx.doi.org/10.1136/ adc.65.3.308

17. Nielsen KG, Auk IL, Bojsen K, Ifversen M, Klug B, Bisgaard H. Clinical effect of Diskus dry-powder inhaler at low and high inspiratory flow rates in asthmatic children. Eur Respir J 1998;11:350-4. http://dx.doi.org/10.1183/09031936.98.11020350

18. Koskela T, Malmström K, Sairanen U, Peltol, S, Keski-Karhu J, Silvasti M. Efficacy of salbutamol via Easyhaler unaffected by low inspiratory flow. Respir Med 2000;94:1229-33. http://dx.doi.org/10.1053/rmed.2000.0959

19. Newman SP, Pitcairn GR, Adkin DA, Vidgren MT, Silvasti M. Comparison of beclomethasone dipropionate delivery by Easyhaler dry powder inhaler and pMDI plus large volume spacer. J Aerosol Med 2001;14:217-25. http://dx.doi.org/10.1089/08942680152484144

20. Thomas M, Price D, Chrystyn H, Lloyd A, Williams AE, von Ziegenwich J. Inhaled corticosteroids for asthma: Impact of practice-level device switching on asthma control. BMC Pulm Med 2009;9:1. http://dx.doi.org/10.1186/1471-2466-9-1

21. Pedersen S, Dubus JC, Crompton GK; ADM IT Working Group. The ADMIT series. Issues in inhalation therapy. (5) Inhaler selection in children with asthma. Prim Care Respir J 2010;19:209-16. http://dx.doi.org/10.4104/pcrj.2010.00043

22. Al-Showair RAM, Tarsin WY, Pearson SB, Chrystyn H. Can all patients with COPD use the correct inhalation flow with all inhalers and does training help? Respir Med 2007;101:2395-401. http://dx.doi.org/10.1016/j.rmed.2007.06.008

23. Broeders ME, Molema J, Hopp WC, Vermue NA, Folgering HT. The course of inhalation profiles during an exacerbation of obstructive lung disease. Respir Med 2004;98:1173-9. http://dx.doi.org/10.1016/j.rmed.2004.04.010

24. Clark A. Effect of powder inhaler resistance upon inspiratory profiles in health and disease. In: Byron PR, Dalby RN, Farr SJ, eds. Respiratory Drug Delivery IV. Volume 1.
Buffalo Grove, IL, USA: Interpharm Press, 1994, p.117-23.

25. De Boer AH, Bolhuis GK, Gjaltema D, Hagedoorn P. Inhalation characteristics and their effect on the in-vitro drug delivery from dry powder inhalers. Int J Pharm 1997;153:67-77. http://dx.doi.org/10.1016/S0378-5173(97)00097-5

26. Chrystyn H, Price D. What you need to know about inhalers and how to use them. Prescriber 2009; Issue 12: 47-52.

27. Broeders ME, M olema J, Vermue NA, Folgering HT. Peak inspiratory flow rate and the slope of the inhalation profiles in dry powder inhalers. Eur Respir J 2001;18:780-3. http://dx.doi.org/10.1183/09031936.01.00240301

28. Lavorini F, Levy ML, Corrigan C, Crompton G; ADM IT Working Group. The ADMIT series. Issues in inhalation therapy. (6) Training tools for inhalation devices. Prim Care Respir J 2010;19:335-41. http://dx.doi.org/10.4104/pcrj.2010.00065

29. Alaboud A, Assi $\mathrm{KH}$, Chrystyn $\mathrm{H}$. In vitro characterization of the emitted dose from the Foradil aerolizer to identify the influence of inhalation flow, inhalation volume and the number of inhalations per dose. In: Dalby RN, Byron PR, Peart J, Suman JD, Young PM, eds. Respiratory Drug Delivery 2010. Volume 3. Richmond, VA, USA: Davis Healthcare International Publishing, Virginia Commonwealth University, 2010, p.803-6.

30. Kamin WES, Genz T, Roeder S, Scheuch G, Trammer T, Juenemann R, Cloes RM. Mass output and particle size distribution of glucocorticosteroids from different inhalation devices depending on various inspiratory parameters. J Aerosol Med 2002;15:65-73. http://dx.doi.org/10.1089/08942680252908593

31. Derom E, Strandgarden K, Schelfhout V, Borgstrom L, Pauwels R. Lung deposition and efficacy of inhaled formoterol in patients with moderate to severe COPD. Respir Med 2007;101:1931-41. http://dx.doi.org/10.1016/j.rmed.2007.04.013

32. Hindle $M$, Newton DAG, Chrystyn $H$. Investigations of an optimal inhaler technique using urinary salbutamol excretion as a measure of relative bioavailability to the lung. Thorax 1993;48:607-11. http://dx.doi.org/10.1136/thx.48.6.607

33. Meakin BJ, Cainey JM, Woodcock PM. Simulated 'in-use' and 'mis-use' aspects of the delivery of terbutaline sulphate from Bricanyl Turbohaler ${ }^{T M}$ dry powder inhalers. Int J Pharm 1995;119:103-08. http://dx.doi.org/10.1016/0378-5173(94)00388-L

34. Lavorini $F$, Corbetta $L$, on behalf of the Aerosol Drug Management Improvement Team (ADMIT). Achieving asthma control: the key role of inhalers. Breathe 2008;5:121-31.

35. van der Palen J, Klein JJ, van Herwaarden CL, Zielhuis GA, Seydel ER. Multiple inhalers confuse asthma patients. Eur Respir J 1999;14:1034-7. http://dx.doi.org/ 10.1183/09031936.99.14510349

\section{Available online at http://w ww.thepcrj.org}

